against other breakers are being arranged. Surveys in factories have been specially directed to the investigation of yarn-winding processes and of humidification. In sizing, promising results have been obtained in trials of a new brushing unit on a slashing machine, and fundamental work on the relationship between yarn and cloth has continued. Methods of bleaching linen goods have been evolved which indicate that still higher quality can be achieved.

\section{Fluorosis and Pollution}

REPLYING to an adjournment debate in the House of Commons on May 14, initiated by Dr. Barnett Stross, of Stoke-on-Trent, who pressed for the instigation of a local inquiry into the incidence of fluorosis due to atmospheric pollution, the Parliamentary Secretary to the Ministry of Health, Miss Patricia Hornsby-Smith, said that, on the evidence available, neither the Ministry nor the Medical Research Council believes that an inquiry in that area is essential. The wider problem of fluorine would, however, have to be one of the items covered in the investigations now being undertaken by the Minister of Housing and Local Government into air pollution, as particularly applied to industrial areas. With regard to the question of fluorine in water supplies, Miss Hornsby-Smith said she did not wish to make any comment pending a full study of the report of the British mission to North America inquiring into this question.

\section{$X$-Rays accompanying $\mu$-Meson Capture}

INTERESTING observations on the X-rays accompanying $\mu$-meson capture by nuclei are reported through the rather unusual channel of a "press release' from Columbia University. Negative $\mu$-mesons brought to rest in matter eventually disappear, either by spontaneous decay or by nuclear capture. In either case the process takes place in two fairly distinct steps. First the meson is attracted into quantum orbits around a nucleus, to which it is bound by the electrical forces. During this stage it emits' X-rays or ejects atomic electrons as it jumps successively from one quantum state to another, until it reaches the state of lowest energy. Disintegration or capture then occurs. The orbits are like those of the interior atomic electrons, but about two hundred times smaller in linear dimensions (a consequence of the greater mass of the meson). The energy of the $\mathrm{X}$-rays is similarly some two hundred times greater than the corresponding atomic $\mathrm{X}$-rays. Rainwater and Fitch have observed these $\mathrm{X}$-rays and made precise determinations of their energies. Since the deepest meson orbit and the nucleus are comparable in size for a beavy nucleus, the energy of the former is strongly influenced by the way in which electric charge is distributed in the nucleus. From their measurements Rainwater and Fitch conclude that the charge is considerably more concentrated towards the centre than had been previously believed. They deduce an effective radius 15 per cent smaller than is yielded by such methods as nuclear binding energies or neutron scattering.

Industrial Application of Aerodynamic Techniques

A NUMBer of special applications of aerodynamic equipment and techniques which have been used in the course of investigations in the Aerodynamics Division of the National Physical Laboratory, Teddington, are described in a little handbook entitled
"The Industrial Application of Aerodynamic Techniques" (National Physical Laboratory: Notes on Applied Science No. 2. Pp. 37. London: H.M.S.O., 1952. 3s.6d. net). It is often impossible to use the wind tunnel for the solution of problems of fluid motion which arise outside the aircraft industry. Dr. R. A. Frazer's work on the aerodynamic stability of suspension bridges is an exception, and the model tests done for the Severn Bridge project are described. Civil and mechanical engineers will deduce without surprise that there are few industrial applications of text-book aerodynamics. This is, of course, true; but one feels that opportunity has been lost by conveying a too sober estimate of the possibilities of applying aerodynamic experience to industrial practice.

\section{Kodaikanal Observatory: Report for 1950}

THE report for 1950 of the Kodaikanal Observatory (pp. 8; Delhi : Manager of Publications, 1951; 7 annas or $8 d$.) deals chiefly with the astronomical work there, though short references are given to geomagnetic observations and seismology; the meteorological data appear in the Indian Weather Review, and the administrative details in the annual report of the Indian Meteorological Department. A list of the forty-seven instruments in use is given and also a brief account of the constructional works relating to development projects, including the construction of a dome for housing a $20-\mathrm{in}$. Grubb reflector and of buildings for an ionosphere laboratory. There has been a considerable amount of co-operation with other observatories-the Solar Physics Observatory, Cambridge, the Royal Greenwich Observatory, the Meudon Observatory, and the Mt. Wilson Observatory - with which there has been exchange of spectroheliograms, photoheliograms; quarterly statements of solar flares, etc. During 1950 the Government of India granted two research scholarships in astronomy tenable for three years at the Kodaikanal Observatory.

\section{Beit Memorial Fellowships for Medical Research}

BEIT Memorial Fellowships for medical research have been awarded as follows. Fourth Year Fellowship ( $£ 735$ a year): Dr. Joan E. Keilin (University of Cambridge), to investigate, at the Departmant of Biochemistry, Cambridge, the catalytic activity of hæmatin compounds and the effect of oxidizing agents on hæmatins, the properties of purified helicorubin and its relationship to other natural hæmoproteins, and the reactions of hæmatins with proteins and their constituent amino-acids and peptides, with the aim of gaining more information on the structure of the hæmoglobin molecule, the link between the hæm and globin and the use of hæmatin as a reagent for the study of other biologically important proteins. Junior Fellowships ( $£ 630$ a year) : J. R. Cannon (University of Sydney), to continue at the University Chemical Laboratories, Cambridge, the study of the chemical structure of vitamin $\mathrm{B}_{12} ;$ G. P. Lewis (University of Wales), to study, at the National Institute for Medical Research, Mill Hill, London, the relationship between structure and action of drugs, particularly of antagonists of histamine, acetylcholine, adrenaline and nor-adrenaline, in order to examine how far ionization of a drug or its receptor modifies drug action; W. L. Nicholas (University of Liverpool), to investigate, at the Department of Zoology, Liverpool, the determination of the growth requirements of free-living and parasitic nematodes in chemically 